\title{
Fully non-inductive plasma start-up with lower-hybrid waves using the outboard-launch and top-launch antennas on the TST-2 spherical tokamak
}

\author{
Naoto Tsujii ${ }^{1,}$, Yuichi Takase ${ }^{1}$, Akira Ejiri ${ }^{1}$, Takahiro Shinya ${ }^{2}$, Satoru Yajima ${ }^{1}$, Hibiki Yamazaki $^{1}$, Hiro Togashi $^{1}$, \\ Charles P. Moeller ${ }^{3}$, Benedikt Roidl $^{1}$, Wataru Takahashi ${ }^{1}$, Kazuya Toida ${ }^{1}$, and Yusuke Yoshida ${ }^{1}$ \\ ${ }^{1}$ The University of Tokyo, Kashiwa 277-8561, Japan \\ ${ }^{2}$ National Institute for Quantum and Radiological Science and Technology, Rokkasho 039-3212, Japan \\ ${ }^{3}$ General Atomics, San Diego, CA 92186, USA
}

\begin{abstract}
Removal of the central solenoid is essential to realize an economical spherical tokamak fusion reactor, but non-inductive plasma start-up is a challenge. On the TST-2 spherical tokamak, non-inductive plasma start-up using lower-hybrid ( $\mathrm{LH})$ waves has been investigated. Using the capacitively-coupled combline (CCC) antenna installed at the outboard midplane, fully non-inductive plasma current ramp-up up to a quarter of that of the typical Ohmic discharges has been achieved. Although it was desirable to keep the density low during the plasma current ramp-up to avoid the LH density limit, it was recognized that there was a maximum current density that could be carried by a given electron density. Since the density needed to increase as the plasma current was ramped-up, the achievable plasma current was limited by the maximum operational toroidal field of TST-2. The top-launch CCC antenna was installed to access higher density with up-shift of the parallel index of refraction. Numerical analysis of LH current drive with the outboard-launch and top-launch antennas was performed and the results were qualitatively consistent with the experimental observations.
\end{abstract}

\section{Introduction}

Spherical tokamaks (STs) are able to operate at high beta [1] and have the potential to realize an economical fusion reactor with low magnetic field. However, it is essential that the central solenoid (CS) be removed to achieve the optimum performance [2-3]. Steady-state plasma current can be carried mostly by the bootstrap current with small assistance from neutral beam current drive [2]. On the other hand, establishment of an efficient and reliable non-inductive plasma start-up method is an active area of research [4-11]. Since neutral beam current drive requires finite plasma current for confinement of fast ions, plasma current ramp-up from no current needs to be performed by other means such as coaxial helicity injection [4] or radio frequency (RF) waves [5-11].

Lower-hybrid (LH) current drive has the highest efficiency among all the known RF current drive methods under optimized conditions. Non-inductive plasma current ramp-up using LH waves has been demonstrated on JT-60U [12]. Formation of an advanced tokamak by switch-over to neutral beam injection has also been observed [12]. The possibility of plasma startup with LH waves on STs has been investigated on TST2 [8-11]. Formation of stable ST equilibria only with LH current drive has been demonstrated [8]. Although there is no fundamental reason to prohibit application of $\mathrm{LH}$ waves to STs, operation at high density is more challenging than conventional tokamaks due to the lower toroidal field. For parameters of present day STs, the parallel index of refraction of the LH waves needs to be relatively large and high current drive efficiency observed in conventional tokamaks [13-15] may be hard to realize. However, future ST reactors [2-3] will have a toroidal field of a few tesla, and efficient current drive can be possible using LH waves especially for plasma start-up when the density is low.

A greater concern may be the uncertainty of the impact of the strong toroidicity of the low-aspect ratio machines. The strong gradient of the toroidal field causes large up-shift or down-shift of the parallel index of refraction, and the wave propagation and damping are strongly altered from what they would be in the absence of up/down-shift. Although difference in the wave propagation for different launch scenarios are qualitatively similar between STs and conventional tokamaks, application of unconventional scenarios such as inboard-launch, top-launch, etc., will likely have greater impact on the current drive efficiency on STs. Therefore, testing different launch scenarios on ST is also a good code validation exercise for the predicted effectiveness of those unique launch scenarios. In this paper, we present our recent numerical analysis of the LH driven fully non-inductive discharges on TST-2.

* Corresponding author: tsujii@.k.u-tokyo.ac.jp 


\section{Experimental setup}

TST-2 is a spherical tokamak $\left(\mathrm{R}_{0}=0.36 \mathrm{~m}, \mathrm{a}=0.23 \mathrm{~m}\right.$, $\left.B_{\mathrm{t} 0}<0.3 \mathrm{~T}, I_{\mathrm{p}}<120 \mathrm{kA}\right)$ [16] located at the University of Tokyo in Japan. The magnets are powered by capacitor banks and the on axis toroidal field is limited to $0.16 \mathrm{~T}$ for LH start-up discharges due to longer pulse length. The maximum plasma current achieved for LH start-up discharges is $25 \mathrm{kA}$ which is about a quarter of that of the typical Ohmic discharges.

Four $100 \mathrm{~kW}$ sources at $200 \mathrm{MHz}$ are available for LH current drive. Presently, two capacitively-coupled combline (CCC) antennas are installed [9, 11]. Compared to the previous antennas, the $\mathrm{CCC}$ antennas have good plasma coupling, directional wavenumber spectrum, and high power handling capability, so that most of the available source power can be delivered to the plasma at the desired spectral range. The outboardlaunch antenna has a peak parallel index of refraction $n_{\|}$ of 6.0 and $200 \mathrm{~kW}$ source power. The top-launch antenna has a peak $n_{\|}$of 4.7 at the center of the straps and $100 \mathrm{~kW}$ source power. The $n_{\|}$needs to be greater than $\sim 3$ for core accessibility. Absorption on the bulk (thermal) distribution occurs at about $n_{\|}=20$ for the present TST-2 temperature. The highest plasma current was achieved using the outboard-launch CCC antenna.

A $5 \mathrm{~kW}$ electron cyclotron heating $(\mathrm{ECH})$ system at $2.45 \mathrm{GHz}$ is also available for reliable breakdown of the plasma. ECH is turned off during the plasma current ramp-up for the results shown in this paper.

\section{Numerical simulations}

A ray-tracing code GENRAY [17] and a Fokker-Planck solver CQL3D [18] was used to simulate LH current drive in this work. The ray trajectories calculated in GENRAY are given to CQL3D for self-consistent simulation of LH wave damping and the electron distribution function. CQL3D solves the bounceaveraged Fokker-Planck equation in the limit of zero orbit width (electrons tied to the flux surfaces). The electron distribution function is evolved in time in the presence of $\mathrm{LH}$ wave quasilinear diffusion until it reaches a near steady-state under the experimentally given density, temperature and current profiles. In LH current drive experiments, waves with highly superthermal phase velocity are usually launched for efficiency. The absorption is weak at LH power turn on, but the fast electron plateau quickly develops, resulting in much stronger absorption and efficient plasma current sustainment. Therefore, it is essential that the wave damping and evolution of the electron distribution function be simulated self-consistently.

\section{LH start-up experimental results}

\subsection{Outboard-launch experimental results}

Figure 1 shows the time evolution of the parameters of a typical non-inductive discharge on TST-2 driven solely
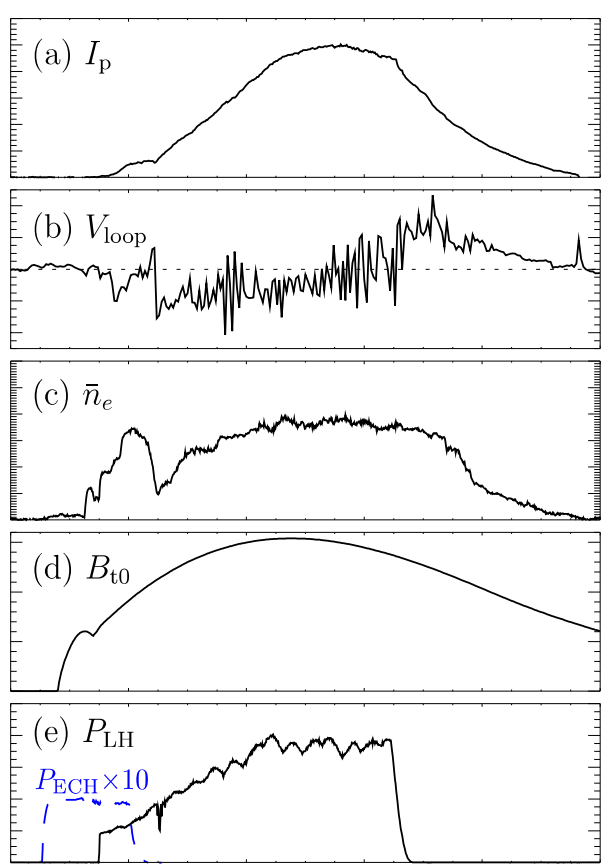

Fig. 1. Time traces of a fully non-inductive plasma start-up discharge by LH waves. The plotted parameters are (a) plasma current, (b) loop voltage, (c) line-averaged electron density, (d) toroidal field, and (e) LH power. ECH power $(\times 10)$ is also shown with the blue dashed curve.

by LH waves. The plasma current reaches a maximum of $25 \mathrm{kA}$ about $40 \mathrm{~ms}$ after the $\mathrm{LH}$ power turn on, and sustained for about $10 \mathrm{~ms}$. Note the negative loop voltage (b) which indicates that this is fully noninductive plasma current ramp-up. The line-averaged density is about $4 \times 10^{17} \mathrm{~m}^{-3}$ which is only a few percent of the typical TST-2 Ohmic discharges. The maximum on axis toroidal field is $0.16 \mathrm{~T}$ for non-inductive discharges and peaks at about $35 \mathrm{~ms}$ after the LH power turn on. The LH power required to sustain this discharge is about $80 \mathrm{~kW}$ (e) which is less than half of the available source power. It was not possible to increase the plasma current by increasing the LH power. This point will be discussed further later.

The electron density and temperature profiles were measured by the Thomson scattering diagnostic (Fig. 2). Because of the low density, the same discharge was repeated and the measurements were ensemble averaged to bring the Thomson scattering signal out of the noise floor [19]. In fully non-inductive plasma current rampup, current holes due to back EMF with little particle and energy confinement have been observed elsewhere [20]. The density and temperature profiles were flat inside the current hole (a "box-type" profile) in these discharges. This does not seem to be the case for TST-2 since the density profile is peaked. The temperature profile is hollow, indicating that the LH power is deposited near the edge. This seems to indicate that there is a substantial radially inward transport of the current density.

The plasma current at the flat-top of the discharge is plotted versus the line-averaged density in Fig. 3. The 


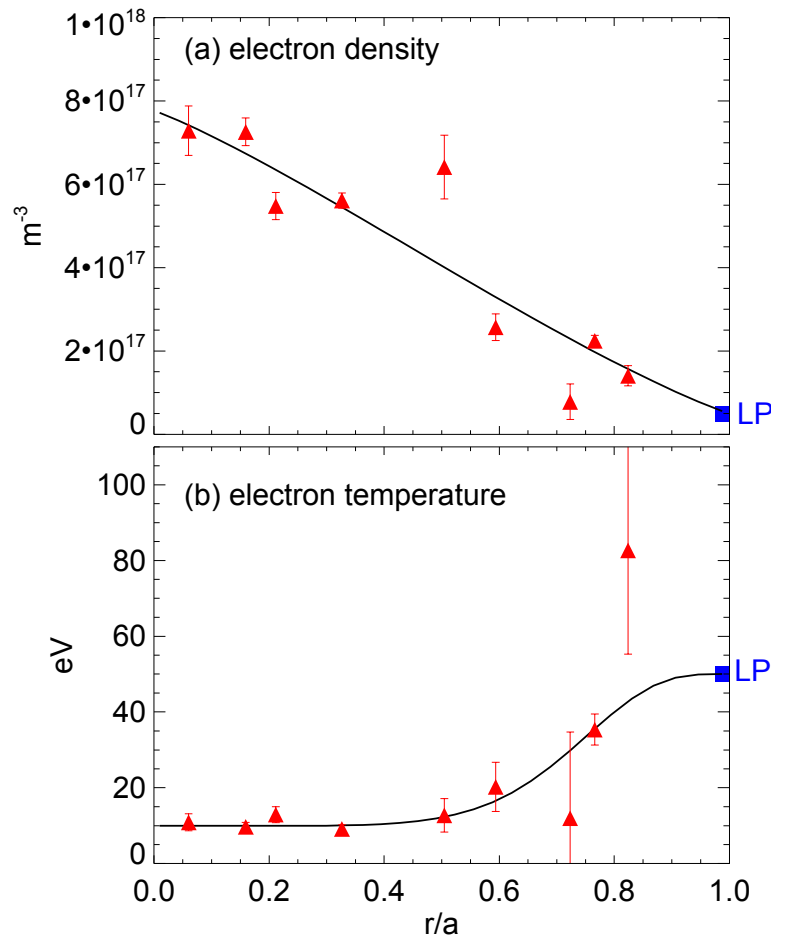

Fig. 2. Density (a) and temperature (b) profiles measured by the Thomson scattering diagnostic plotted versus the normalized minor radius (red triangles). The Langmuir probe measurement is also shown with the blue squares. Fits to the measured points used in the simulation are shown with the solid curves.

plasma current increased at higher toroidal field, which is consistent with the improved accessibility of low $n_{\|}$ component of the LH waves. On the other hand, the achievable plasma current decreased slightly with decreasing density, which was the opposite of what was expected from previous LH current drive experiments in conventional tokamaks [13-15]. This is a potential limitation for using LH on STs, since operation at as low density as possible is desired. If the density needs to increase with the plasma current, the plasma current will be limited at the mode conversion limit under fixed toroidal field.

To understand the observed parametric dependence of the plasma current, LH current drive was modelled using the GENRAY-CQL3D simulation. The density was scanned in the simulation for discharges obtained in TST-2 for three different values of toroidal fields. The results are shown in Fig. 4. In the simulation, the scrapeoff-layer (SOL) was assumed to have a constant density of $5 \times 10^{16} \mathrm{~m}^{-3}$ and a constant temperature of $50 \mathrm{eV}$ which are the typical values measured by the Langmuir probe installed on the limiter. The temperature profile was fixed to the profile shown in Fig. 2 (b) since little variation of temperature was observed in the experiment. About $60 \%$ of the power was predicted to be absorbed through electron Landau damping in the SOL. A smaller fraction of power $(<10 \%)$ was absorbed in the cold core of the plasma. The simulated total driven current

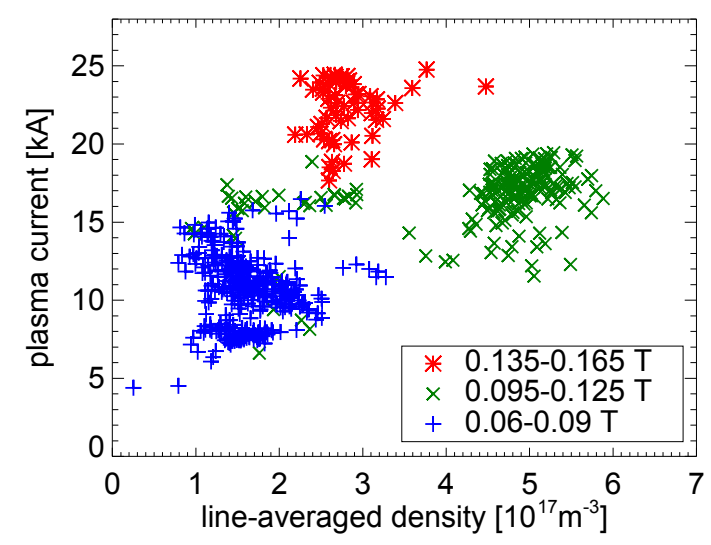

Fig. 3. The observed plasma current versus the line-averaged density for three different ranges of magnetic field; red asterisks: $0.135-0.165 \mathrm{~T}$, green crosses: $0.095-0.125 \mathrm{~T}$, blue plus symbols: 0.06-0.09 T.

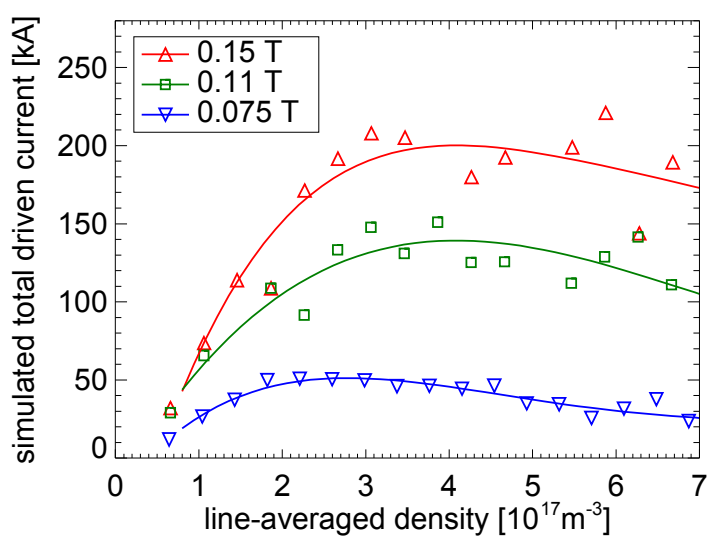

Fig. 4. Simulated total driven current versus the line-averaged density for three different values of toroidal field; red upward triangles: $0.15 \mathrm{~T}$, green squares: $0.11 \mathrm{~T}$, blue downward triangles: $0.075 \mathrm{~T}$. The solid curves are the fits to the simulated points.

decreased at higher density due to deterioration of the LH wave accessibility to the core as was expected.

On the other hand, reduction of the driven current at low density is not usually seen. The simulated parallel electron distribution function at $0.11 \mathrm{~T}$ together with the radial profile of the driven current is shown in Fig. 5 for the optimum and the low density (see Fig. 4, green square symbols). In LH current drive, the wave generates a plateau in the electron parallel velocity distribution function which carries the current [21]. The low-velocity end of the plateau is 2-3 times the thermal velocity below which the absorption is strong and no wave power exists. The high-velocity end, i.e., the maximum phase velocity (corresponding to the minimum $n_{\|}$) of the LH waves, is more important regarding the maximum current density. This limit corresponds to the wave accessibility limit determined by the mode conversion condition. For the cases shown in Fig. 5, it can be seen that the fast electron plateau is completely flat and saturated. Under this condition, the plateau level 

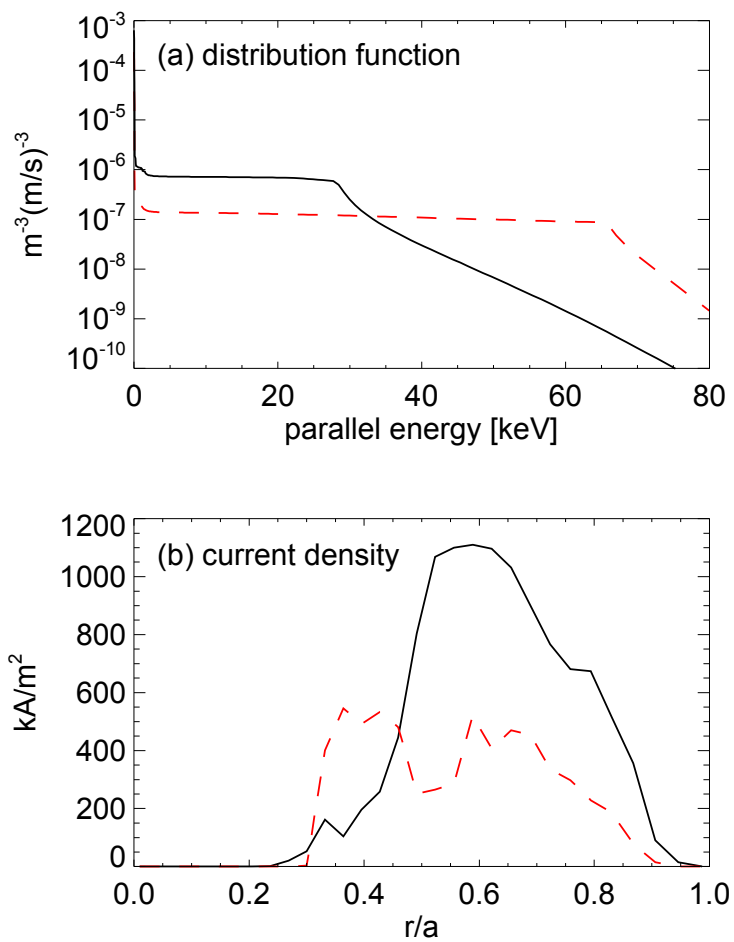

Fig. 5. The parallel electron distribution function at $\mathrm{r} / \mathrm{a}=0.59$ (a) and the radial profile of the driven current (b) at the optimum and the low density, $0.11 \mathrm{~T}$. Black solid curves: $3.9 \times 10^{17} \mathrm{~m}^{-3}$, red dashed curves: $1.1 \times 10^{17} \mathrm{~m}^{-3}$.

decreases in proportion to the density. Although wave spectrum with higher phase velocity become accessible at lower density so that the electrons are accelerated to higher energy, the net change is a factor of two reduction in the driven current density (Fig. 5 (b)). That is, our density is so low that the current density is at its theoretical maximum which is proportional to the electron density (Equation (4) in [21]). Increasing the LH power does not help increase the driven current under this circumstance since the fast electron plateau is already saturated.

In such a low density regime, there is an optimum density for current drive determined by the balance of a sufficient current carrier (electron) density and good LH wave accessibility to the plasma core which requires low density. According to the GENRAY-CQL3D simulation, the optimum density is $2-6 \times 10^{17} \mathrm{~m}^{-3}$ for $B_{\mathrm{t} 0}=0.11 \mathrm{~T}$ (Fig. 4, green squares). This is consistent with the operational density range in the actual experiment (Fig. 3 , green crosses). The simulated total driven current starts to fall off as a function of density above $4.0 \times 10^{17}$ $\mathrm{m}^{-3}$ at $0.11 \mathrm{~T}$. The fall-off density decreases to $2.5 \times 10^{17}$ $\mathrm{m}^{-3}$ at $0.075 \mathrm{~T}$ due to worse accessibility. The operational density range in the actual experiment also decreases by a factor of two from $\sim 0.11 \mathrm{~T}$ to $\sim 0.075 \mathrm{~T}$, consistently with the simulation.

Appreciable increase in the total driven current was predicted when the toroidal field was increased from $0.11 \mathrm{~T}$ to $0.15 \mathrm{~T}$, which was observed in the experiment as well. On the other hand, the predicted increase in the driven current from $0.076 \mathrm{~T}$ to $0.11 \mathrm{~T}$ was not observed in the experiment. In the previous experiments on TST2 , the plasma current ramp-up up to about $4 \mathrm{kA}$ was possible with counter-current drive LH antenna phasing [8], indicating substantial contribution of the bootstrap current for the lower current discharges. Since bootstrap current is not taken into account in the present analysis, this may explain the discrepancy.

The overall value of the simulated driven current (Fig. 4) was up to an order of magnitude greater than the overall plasma current observed in the actual experiment (Fig. 3). The typical fast electron energy for the LH driven non-inductive discharges on TST-2 is about 20 $\mathrm{keV}$ (Fig. 5 (a)). At the present level of plasma current, the poloidal field is at most $\sim 10 \mathrm{mT}$, and the trapped particle orbit widths for the $20 \mathrm{keV}$ electrons are $0.07 \mathrm{~m}$, which is one-third of the plasma minor radius. Under such a condition, acceleration of electrons through RF quasilinear diffusion is balanced not only by the collisional slowing down, but also with collisional radial diffusion (neoclassical diffusion). Additional losses of fast electrons associated with the radial diffusion will likely lead to smaller driven current and may bring the predicted value closer to the experimental observation. Either Monte-Carlo approach [22] or Fokker-Planck solver valid for finite orbit width [23] is necessary to account for these effects and also to evaluate bootstrap current in the presence of fast electrons.

\subsection{Top-launch experimental results}

For further current ramp-up under the present toroidal field, we have developed a top-launch antenna [11]. LH waves launched from the top of the plasma undergo upshift of the parallel index of refraction and higher and higher density becomes accessible as the waves propagate radially inward. Although current drive efficiency decreases when the parallel index of refraction increases, it is hoped that higher operational density will allow us to achieve higher plasma current. When the rays are launched from the outboard side, they diffract outward at higher density. On the other hand, the ray trajectory for the waves launched from the top of the plasma barely changes (Fig. 5 in [11]).

Non-inductive plasma start-up experiment using the top-launch antenna started at the beginning of 2016 [11]. Although we were able to develop an operational scenario using only the top-launch antenna, plasma current ramp-up was most successful when the outboardlaunch antenna was used together with the top-launch antenna. For demonstration of the improvement of the wave accessibility, we show the comparison of the discharges with and without the top-launch antenna power during the ramp-down of the toroidal field since it was difficult to perform accurate density control with the present system implemented on TST-2. The time traces of the parameters are shown in Fig. 6. After the plasma current reached a flat-top value of about $10 \mathrm{kA}$, the density (b) and the vertical field (d) was kept steady while the toroidal field (c) ramped down. The black curves show the traces of the discharge with only the outboard-launch antenna power of $60 \mathrm{~kW}$. The red 

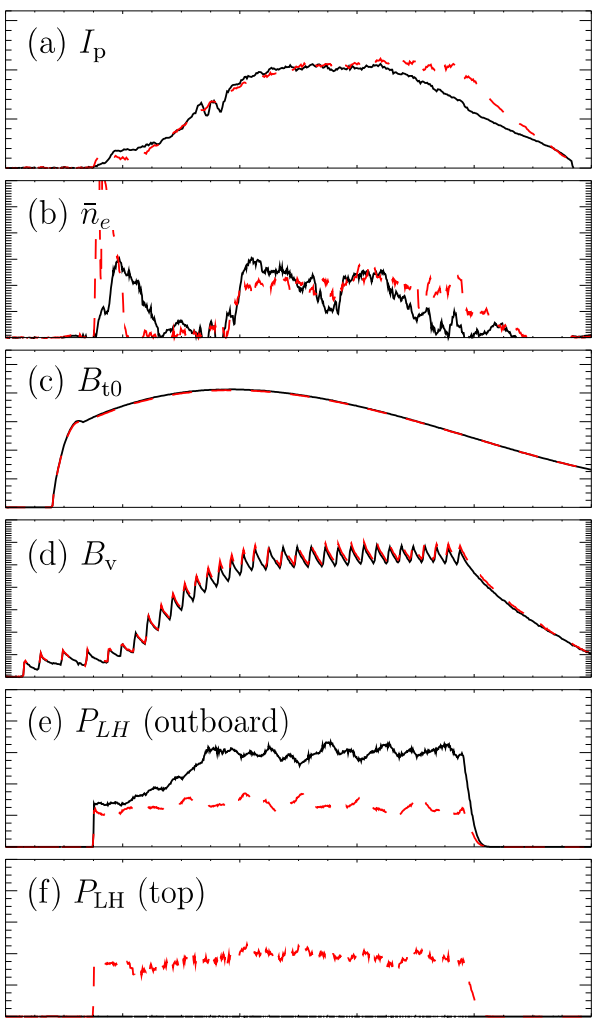

Fig. 6. Time traces of the fully non-inductive plasma start-up discharge by LH wave with only the outboard-launch antenna (black solid curves) and with both the top-launch and the outboard-launch antennas (red dashed curves). The plotted parameters are (a) plasma current, (b) line-averaged electron density, (c) toroidal field, (d) vertical field, (e) outboard-launch antenna power, and (f) top-launch antenna power.

curves show those with the same total LH power but half the LH power replaced by the top-launch antenna power.

The measured plasma current (a) is plotted versus the toroidal field in Fig. 7. When only the outboard-launch antenna was used, plasma current starts to fall off below 0.067 T. On the other hand, when the top-launch antenna was used together with the outboard-launch antenna, the plasma current was sustained until the end of the discharge despite the same total LH power. The simulated total driven current for the two discharges is shown in Fig. 8. The simulated driven current with the top-launch antenna was always higher than that at 0.067 $\mathrm{T}$ with the outboard-launch antenna only, consistently with the experimental observation. Note that the simulated total driven current shows how much current can potentially be driven for the given parameters. In the actual experiment, the plasma current is limited by the vertical field and is held constant as long as sufficient current drive power is provided.

\section{Optimization of LH current drive with poloidal phasing}

The dependence of the experimentally observed driven current on the toroidal field and different launch

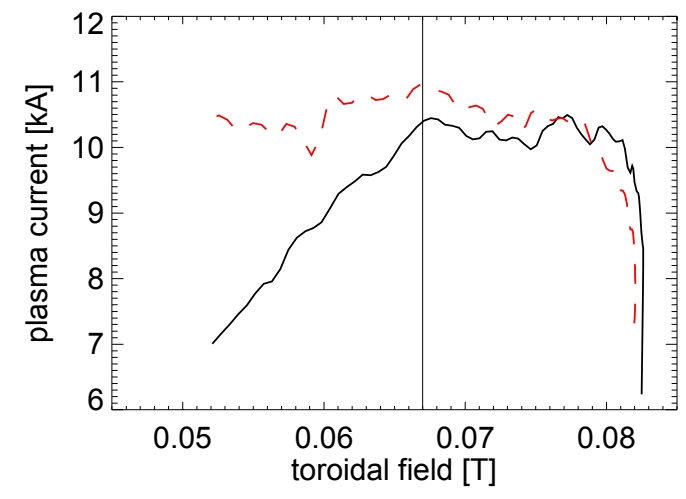

Fig. 7. The measured plasma current plotted versus the toroidal field. Black solid curve: outboard-launch only, red dashed curve: both top-launch and outboard-launch.

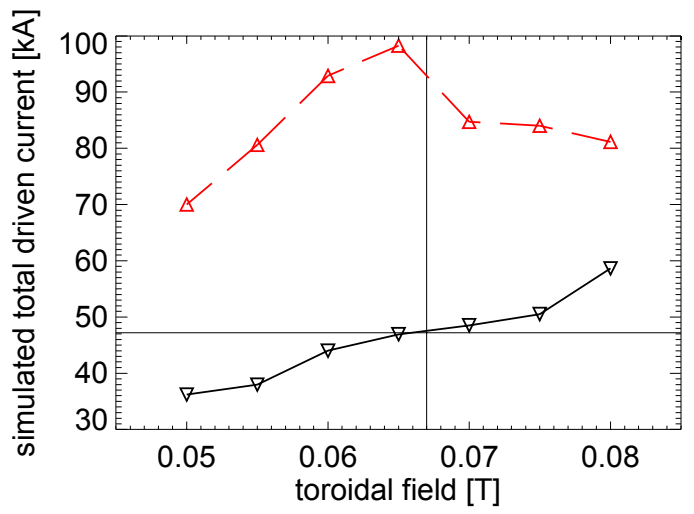

Fig. 8. The simulated total driven current plotted versus the toroidal field. Black solid curve: outboard-launch only, red dashed curve: both top-launch and outboard-launch.

locations could be qualitatively reproduced with the present GENRAY-CQL3D model. If the present plasma current limit is indeed do to saturation of the fast electron plateau, higher current could be obtained only by optimizing the wave spectrum in the core plasma.

The theoretical maximum current density is achieved when the $n_{\|}$spectrum is such that the parallel phase velocity extends all the way from electron thermal velocity up to the maximum phase velocity given by the mode conversion condition. As mentioned in the introduction, waves with highly superthermal parallel phase velocity are usually launched, and the gap between this velocity and the electron thermal velocity is filled by up-shift of $n_{\|}$as the waves travel in and out of the plasma core. Such a strategy is empirically known to work under the ideal circumstance with minimal SOL losses.

The optimum launch condition can be obtained by targeting the rays such that they mode convert at the location where current drive is desired. The condition was evaluated for TST- 2 by launching the rays at $\mathrm{r} / \mathrm{a}=$ 0.2 on the high-field side midplane with mode conversion condition, and propagating them back in time. This gave a launch location slightly above the midplane with strong poloidal phasing in addition to the toroidal phasing. Fig. 9 shows the comparison of the driven current profile with the present outboard-launch 
antenna phasing (toroidal index of refraction $n_{\text {tor }}=6.1$ ) and with

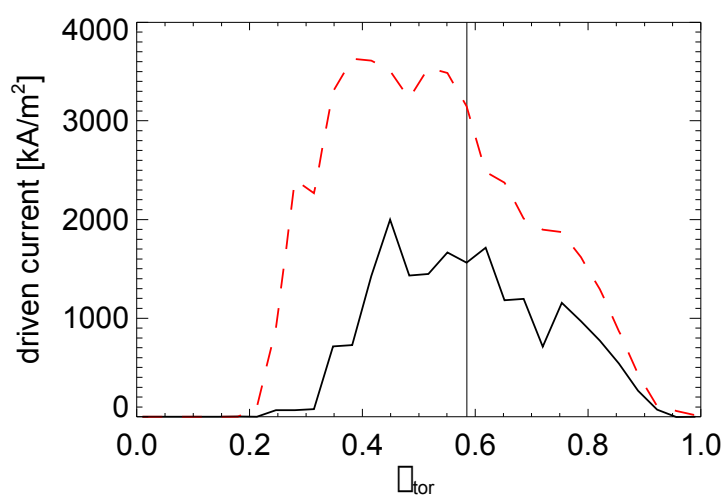

Fig. 9. The simulated total driven current plotted versus the normalized minor radius. Black solid curve: the present outboard-launch antenna, $n_{\text {tor }}=6.1(216 \mathrm{kA})$, red dashed curve: with poloidal phasing, $n_{\text {tor }}=1.8, n_{\text {pol }}=-17(442 \mathrm{kA})$.

strong poloidal phasing ( $n_{\text {tor }}=1.8$ and poloidal index of refraction $n_{\text {pol }}=-17$ ). Increase of the total driven current by a factor of two was predicted. With the poloidal phasing, the launched rays propagate below the midplane while the imposed poloidal phasing is down-shifted and $n_{\|}$is maintained nearly constant until they transform to fast waves around the targeted location. Further analysis is still required to find the optimum launch condition for various toroidal field and density, but imposing certain degree of poloidal phasing appears to have a beneficial impact in general.

\section{Conclusions and future work}

Non-inductive plasma start-up with the LH current drive on a ST has been demonstrated on TST-2. With the newly available electron density and temperature profiles, realistic numerical modeling of LH current drive was performed using the GENRAY-CQL3D simulation. The plasma current was observed to increase slightly with increasing density, which is the trend opposite to what was expected from previous LH current drive experiments in conventional tokamaks. The numerical analysis of the TST-2 discharges showed that the driven current indeed decreases at lower density due to shortage of current carriers (electrons). This parameter regime has never been accessed in other devices which have much higher density and and much lower LH power per electron. When the top-launch antenna was used, the density limit was higher than the discharge driven only by the outboard-launch antenna as was expected theoretically.

For future work, the cause of the overprediction of the simulated driven current needs to be investigated. The present analysis assumes zero electron orbit width. Finite orbit model is probably necessary to evaluate quantitatively accurately the LH driven current and the bootstrap current on TST-2. An improved antenna model may also be necessary to accurately describe the high $n_{\|}$ part of the wave spectrum. The internal current profile is also not accurately evaluated in the experiment. The impact of current profile on the wave propagation needs to be investigated. The MEGA code [24] is being extended to investigate the impact of fast electrons on the MHD equilibrium and instabilities.

Finally, poloidal phasing was theoretically shown to be beneficial for current drive. Feasibility of such an antenna design will be investigated.

This work was supported by JSPS KAKENHI Grant number 21226021, NIFS Collaboration Research Program under NIFSKOCR001, NIFS12KUTR078, and NIFSKNWR001, and Japan/US Cooperation in Fusion Research and Development. Work at General Atomics is supported by US DoE contract DE-AC03-97ER-54411. Collaborations with P. Bonoli, J. Wright, and J. Lee at MIT on GENRAY/CQL3D calculations are gratefully acknowledged.

\section{References}

1. Y.K. Peng, and D. Strickler, Nucl. Fusion 26, 769 (1986)

2. F. Najmabadi, Fusion Eng. Des. 65, 143 (2003)

3. S. Nishio, et al., 20th International Conference on Fusion Energy, FT/P7-35 (2004)

4. R. Raman, et al., Phys. Rev. Lett. 104, 095003 (2010)

5. V.F. Shevchenko, et al., Nucl. Fusion 50, 022004 (2010)

6. M. Uchida, et al., Phys. Rev. Lett. 104, 065001 (2010)

7. H. Miura, et al., Plasma Fus. Res. 10, 3402066 (2015)

8. Y. Takase, et al., Nucl. Fusion 51, 063017 (2011)

9. Y. Takase, et al., Nucl. Fusion 53, 063006 (2013)

10. T. Wakatsuki, et al., Nucl. Fusion 54, 093014 (2014)

11. T. Shinya, et al., Nucl. Fusion 57, 036006 (2017)

12. S. Shiraiwa, et al., Phys. Rev. Lett 92, 035001 (2004)

13. S. Shiraiwa, et al., Nucl. Fusion 53, 113028 (2013)

14. K.K. Kirov, et al., Plasma Phys. Control. Fusion 55, 115008 (2013)

15. M. Goniche, et al., Nucl. Fusion 53, 033010 (2013)

16. Y. Takase, et al., Nucl. Fusion 41, 1543 (2001)

17. A. Smirnov, and R. Harvey, Bull. Am. Phys. Soc. 39, 1626 (1994)

18. R.W. Harvey, and M.G. McCoy, Proceedings of the IAEA Technical Committee Meeting on Advances in Simulation and Modeling of Thermonuclear Plasmas, 489 (1992)

19. H. Togashi, et al., J. Inst. 10, C12020 (2015)

20. T. Fujita, Nucl. Fusion 50, 113001 (2010)

21. N.J. Fisch, Phys. Rev. Lett. 41, 873 (1978)

22. S. Murakami, et al., Nucl. Fusion 46, S425 (2006)

23. Y.V. Petrov, and R.W. Harvey, Plasma Phys. Control. Fusion 58, 115001 (2016) 
24. Y. Todo, and A. Bierwage, Plasma Fus. Res. 9, 3403068 (2014) 